\title{
Dyson's Instability in Lattice Gauge Theory
}

\section{A. Bazavov ${ }^{b}$, A. Denbleyker ${ }^{a}$, Daping Du ${ }^{a}$, Y. Meurice ${ }^{*} a$, A. Velytsky ${ }^{c}$ and Haiyuan Zou ${ }^{a}$}

${ }^{a}$ Department of Physics and Astronomy, The University of Iowa

Iowa City, Iowa 52242, USA

E-mail: alan-denbleyker@uiowa.edu

E-mail: daping-du@uiowa.edu

E-mail: yannick-meurice@uiowa.edu

E-mail: haiyuan-zou@uiowa.edu

${ }^{b}$ Physics Department, University of Arizona

Tucson, AZ 85721, USA

E-mail: bazavov@physics.arizona.edu

${ }^{c}$ Physics Department, Brookhaven National Laboratory

Upton NY 11973, USA

E-mail: vel@bnl.gov

We discuss Dyson's argument that the vacuum is unstable under a change $g^{2} \rightarrow-g^{2}$, in the context of lattice gauge theory. For compact gauge groups, the partition function is well defined at negative $g^{2}$, but the average plaquette $P$ has a discontinuity when $g^{2}$ changes sign. This reflects a change of vacuum rather than a loss of vacuum. In addition, $P$ has poles in the complex $g^{2}$ plane, located at the complex zeros of the partition function (Fisher's zeros). We discuss the relevance of these singularities for lattice perturbation theory. We present new methods to locate Fisher's zeros using numerical values for the density of state in $S U(2)$ and $U(1)$ pure gauge theory. We briefly discuss similar issues for $O(N)$ nonlinear sigma models where the local integrals are also over compact spaces.

The XXVII International Symposium on Lattice Field Theory - LAT2009

July 26-31 2009

Peking University, Beijing, China

\footnotetext{
${ }^{*}$ Speaker.
} 


\section{Introduction}

Dyson instability $[1,2]$ - the catastrophe happening when you change the sign of $e^{2}$ in QED - is often invoked to limit the validity of perturbation theory and justify the factorial growth of the perturbative coefficients. In the functional integral formulation of scalar models, this type of instability is related to large field configurations [3, 4].

For lattice models with compact field integration (nonlinear sigma models over compact manifolds and lattice gauge theories (LGT) with compact groups), the large field problem is in principle absent. For $g^{2}<0$, the partition function is well defined and the change of sign of $g^{2}$ appears as a mere change in vacuum rather than a catastrophic instability. Can this explain the apparent power growth (rather than a factorial growth) observed in perturbative series for the average plaquette $P$ in Refs. $[5,6,7,8]$ ? These series are consistent with the existence of Fisher's zeros (zeros of the partition function in the complex coupling plane) close to the real axis [9]. It seems clear that a complete knowledge of the location of the Fisher's zero would provide a compete understanding of the complex singularities of $P$. The volume dependence of these zeros also provides important information regarding the order of possible transitions or the absence thereof $[10,11]$.

In these proceedings, we report recent results concerning these questions. Dyson instability is reviewed in Sec. 2 in the context of LGT. New "topological" methods to locate Fisher's zeros in $S U(2)$ and $U(1)$ using numerical calculations of the density of states $[12,14]$ are discussed in Sec. 3. Similar questions for $O(N)$ sigma models in the complex t' Hooft coupling plane are briefly discussed in Sec. 4. Details can be found in a recent preprint [15].

\section{Dyson's instability versus compact integration}

Dyson's argument goes as follows [1,2]. Suppose that a physical quantity in QED can be calculated as a perturbative series $F\left(e^{2}\right)=a_{0}+a_{1} e^{2}+\ldots$. If we assume that the series has a finite radius of convergence, then, for $e^{2}$ sufficiently small, we can interpret $F\left(-\left|e^{2}\right|\right)$ as the value of this quantity in a fictitious world where same charge particles attract. But in this fictitious world, every physical state is unstable. So, the radius of convergence is zero. Quoting the author "The argument [...] is lacking in mathematical rigor and in physical precision. It is intended to be suggestive, to serve as a basis for further discussions".

The connection between asymptotic series and the problem of integrating large fields contributions can be understood with this very simple example

$$
\int_{-\infty}^{+\infty} d \phi e^{-\frac{1}{2} \phi^{2}-\lambda \phi^{4}} \neq \sum_{q=0}^{\infty} \frac{(-\lambda)^{q}}{q !} \int_{-\infty}^{+\infty} d \phi e^{-\frac{1}{2} \phi^{2}} \phi^{4 q} .
$$

The sum and the integration have been interchanged illegally. The peak of the integrand of the $q$-th order term of the r.h.s is reached when $\phi^{2}=4 q$. The approximation of $e^{-\lambda \phi^{4}}$ by an expansion of order $q$ in $\lambda \phi^{4}$ is good provided that $\lambda \phi^{4}<<q$, but at the peak of the integrand, $\phi^{4}=16 q^{2}$ and we need $\lambda 16 q^{2}<<q$, which fails for $q$ large enough. On the other hand, if we introduce a field cutoff, as the order increases, at some order, the peak moves outside of the integration range and there is no factorial growth. The general expectation is that for a finite lattice, the partition function $Z$ calculated with a field cutoff is convergent and $\ln (Z)$ has a finite radius of convergence controlled 
by the zeros of the partition function. The field cutoff $\phi_{\max }$ is an optimization parameter fixed using strong coupling [4], for instance.

A fact that is obvious but which importance regarding weak coupling expansions may have been overlooked is that lattice gauge theories with a compact group and nonlinear $O(N)$ sigma models have a build-in large field cutoff. In lattice gauge theory, the group elements associated with the links are integrated with $d U_{l}$ the compact Haar measure. Our notations are as follows: $N_{c}$ is the number of colors, $S=\sum_{\text {plaq. }}\left(1-\left(1 / N_{c}\right) \operatorname{Re} \operatorname{Tr}\left(U_{p}\right)\right)$ and $\beta=2 N_{c} / g^{2}$. The number of plaquettes is denoted $\mathscr{N}_{p} \equiv L^{D} D(D-1) / 2$. The average plaquette: $P(\beta) \equiv\left(1 / \mathscr{N}_{p}\right)\langle S\rangle$ will be our main object of study. The partition function $Z(\beta)$ is the Laplace transform of $n(S)$, the density of states:

$$
Z(\beta)=\int_{0}^{S_{\max }} d S n(S) \mathrm{e}^{-\beta S}
$$

with

$$
n(S)=\prod_{l} \int d U_{l} \delta\left(S-\sum_{p}\left(1-\left(1 / N_{c}\right) \operatorname{Re} \operatorname{Tr}\left(U_{p}\right)\right)\right)
$$

Assuming that $\ln (n(S))$ is extensive we can write

$$
n(S)=\mathrm{e}^{\mathscr{N}_{p} f\left(S / \mathscr{N}_{p}\right)}
$$

It is important to notice that at finite volume, $S_{\max }$ is finite. For instance, $S_{\max }=2 \mathscr{N}_{p}$ for $S U(2 N)$ and $\frac{3}{2} \mathscr{N}_{p}$ for $S U(3)$. In the strong coupling expansion, we expand in power of $\beta: Z=\sum_{n=0}^{\infty} z_{n} \beta^{n}$ with $\left|z_{n}\right|<S_{\text {max }}^{n} / n$ !, so at finite volume, $Z$ is an analytical function, not only on the negative real axis, but over the entire $\beta$ plane.

On the other hand, it is possible to show that for $S U(2 N)$ on even lattices [16]

$$
Z(-\beta)=\mathrm{e}^{2 \beta \mathscr{N}_{p}} Z(\beta) \text {. }
$$

Consequently,

$$
n\left(2 \mathscr{N}_{p}-S\right)=n(S) \text { and } P(\beta)+P(-\beta)=2
$$

Since $\lim _{\beta \rightarrow+\infty} P(\beta)=0, P$ has a discontinuity at $g^{2}=0$ and a regular series for $P$ about $g^{2}=0$ is not possible. However, it does not necessarily mean that the series has factorial growth.

It is useful to consider first the case of a single $S U(2)$ plaquette [17]. In that case, $n(S)=$ $\frac{2}{\pi} \sqrt{S(2-S)}$ (invariant under $S \rightarrow 2-S$ ). The large order of the weak coupling expansion $\beta \rightarrow+\infty$ is determined by the behavior of $n(S)$ near $S=2$, itself probed when $\beta \rightarrow-\infty$ in agreement with the common wisdom that the large order behavior of weak coupling series can be understood in terms of the behavior at small negative coupling. $\sqrt{2-S}$ is then expended about $S=0$ (radius of convergence $=2$ ). This yields the convergent expansion

$$
Z(\beta)=(\beta \pi)^{-3 / 2} 2^{1 / 2} \sum_{l=0}^{\infty}(2 \beta)^{-l} \frac{\Gamma(l+1 / 2)}{l !(1 / 2-l)} \int_{0}^{2 \beta} d t \mathrm{e}^{-t} t^{l+1 / 2}
$$

As expected this is a not a regular series in the sense that the "coefficients" of $\beta^{-l}$ depend on $\beta$, but in a way that is invisible in perturbation theory. The crucial step is to get $\beta$-independent coefficients by neglecting the missing tails of integration.

$$
\int_{0}^{2 \beta} d t \mathrm{e}^{-t} t^{l+1 / 2} \simeq \int_{0}^{\infty} d t \mathrm{e}^{-t} t^{l+1 / 2}+O\left(\mathrm{e}^{-2 \beta}\right)
$$


which in turn creates a factorial growth of the coefficients. The peak of the integrand crosses the boundary near order $2 \beta$. Dropping higher order terms (than order $\simeq 2 \beta$ ) agrees with the rule of thumb (minimizing the first contribution dropped). The non-perturbative part can be fully reconstructed (higher orders + tails) [18]. For $L^{4}$ lattices, the crossing should be near order $2 \beta \mathscr{N}_{p}$. Non-perturbative effects should be explainable by the contributions near $S_{\max }$. We plan to study this question on small lattices.

\section{Fisher's zeros from the density of states}

The poles of $P$ are located at the Fisher's zeros. At finite volume, we expect these zeros to be isolated in the $\beta$ plane. It seems plausible that the zeros will accumulate along lines going through 0 in the $1 / \beta$ plane as they do for Bessel functions. It is possible to use $n(S)$ to calculate $Z$ at complex $\beta$. The calculation of $n(S)$ for $S U(2)$ is discussed in Ref. [12]. Additional checks were made by calculating the first three moments of $n(S)$. For $U(1)$ lattice gauge theory, multicanonical methods relying on the Biased Metropolis-Heatbath Algorithm [13] were used [14]. Using this $U(1)$ density of states, we have calculated the plaquette distribution calculated at fixed $\beta$ and checked that there is an approximately symmetric double peak near $\beta=0.979$ for a $4^{4}$ lattice.

For both $U(1)$ and $S U(2)$ on a $4^{4}$ lattice, the numerical calculation of $Z(\beta)$ with $\operatorname{Im} \beta \sim 0.2$ is difficult because $\beta$ is multiplied by $\mathscr{N}_{p}$ and the integrand oscillate rapidly. A preliminary idea of the distribution of zero can be obtained using semi-classical methods. Using the "color entropy" $f(s)$ defined in Eq. (2.4), the saddle point of the integral is at $s_{0}$ given by solving $f^{\prime}\left(s_{0}\right)=\beta$. $\mathrm{Z}$ becomes a Gaussian integral with correction of order $\sqrt{1 / \mathscr{N}_{p}}$ as long as $\operatorname{Re} f^{\prime \prime}\left(s_{0}\right)<0$. As a Gaussian density of states has no complex zeros [19], it seems clear that zeros should appear in regions of the $\beta$ plane corresponding to regions of the $s$ plane such that $\operatorname{Re} f^{\prime \prime}\left(s_{0}\right)>0$. Using Chebyshev approximations of $f(s)$, we have constructed the boundary $\left(\operatorname{Re} f^{\prime \prime}(s)=0\right)$. The results are shown in Fig. 1. The boundary form narrow tongues ending at a complex zeros of $f^{\prime \prime}$. These complex zeros are then mapped in the $\beta$ plane using $f^{\prime}$. Their number depends on the degree of the polynomial approximation, but the general shape is robust under changes in the degree. It appears that in the case of $S U(2)$ the images in the $\beta$ plane are never on the real axis in contrast to the case of $U(1)$.

New methods have been developed to locate the Fisher zeros $[15,20]$. Given the fact that $Z$ is an entire function in the $\beta$ plane, and that $P=-(d Z / d \beta) / Z$, the worse thing that can happen to $P$ is that $Z$ has a zero of order $k$, say at $\beta_{0}$. Then $(d Z / d \beta) / Z \simeq k /\left(\beta-\beta_{0}\right)$ for $\beta \simeq \beta_{0}$. If we now integrate over a closed contour $C$,

$$
(i 2 \pi)^{-1} \oint_{C} d \beta(d Z / d \beta) / Z=\sum_{k} n_{k}(C)
$$

where $n_{k}(C)$ is the number of zeros of order $k$ inside $C$. This allows us to monitor the accuracy of the calculation. We need to check that in good approximation, the real part is an integer and the imaginary part is zero. This is illustrated in Fig. 2 for a rectangular contour of variable height in the $\beta$ plane. Despite these encouraging results, there remain dependence on the interpolation or fit used to evaluate $f(s)$ numerically. Resolving this issue should allow us to find finite size scaling for the zeros as discussed in Refs. [10, 11]. 

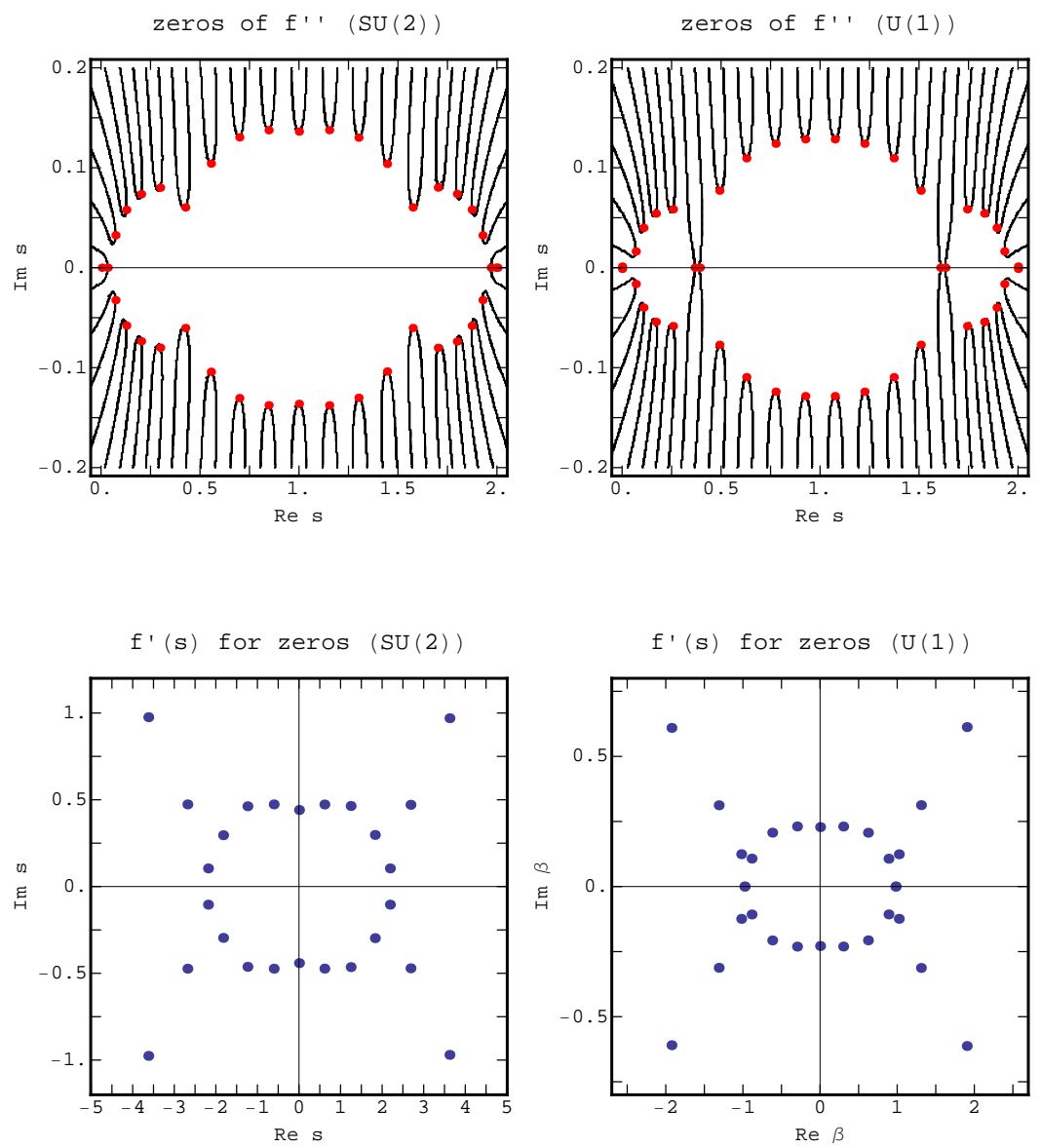

Figure 1: Top: complex zeros and zeros of the real part of $f^{\prime \prime}(s)$ in the complex $s$ plane with 40 Chebyshev polynomials on $4^{4}$ for $S U(2)$ (left) and $U(1)$ (right). Bottom: $f^{\prime}(s)$ evaluated at the complex zeros of $f^{\prime \prime}(s)$ shown on the previous figure for $S U(2)$ (left) and $U(1)$ (right).
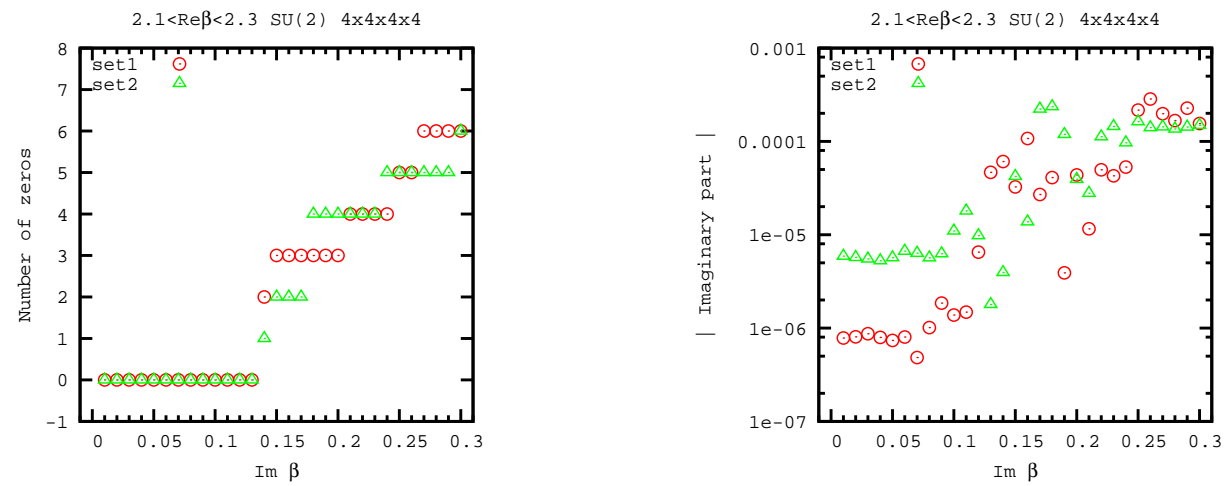

Figure 2: $\operatorname{Re}$ (left) and Im (right) part of $\sum_{k} n_{k}$ defined in Eq. (3.1) for a rectangular contour with $2.1<$ $\operatorname{Re} \beta<2.3$ and $0<\operatorname{Im} \beta<y$ with a variable $y$, for $S U(2)$ on a $4^{4}$ lattice. Two independent numerical values of $n(S)$ were used. 

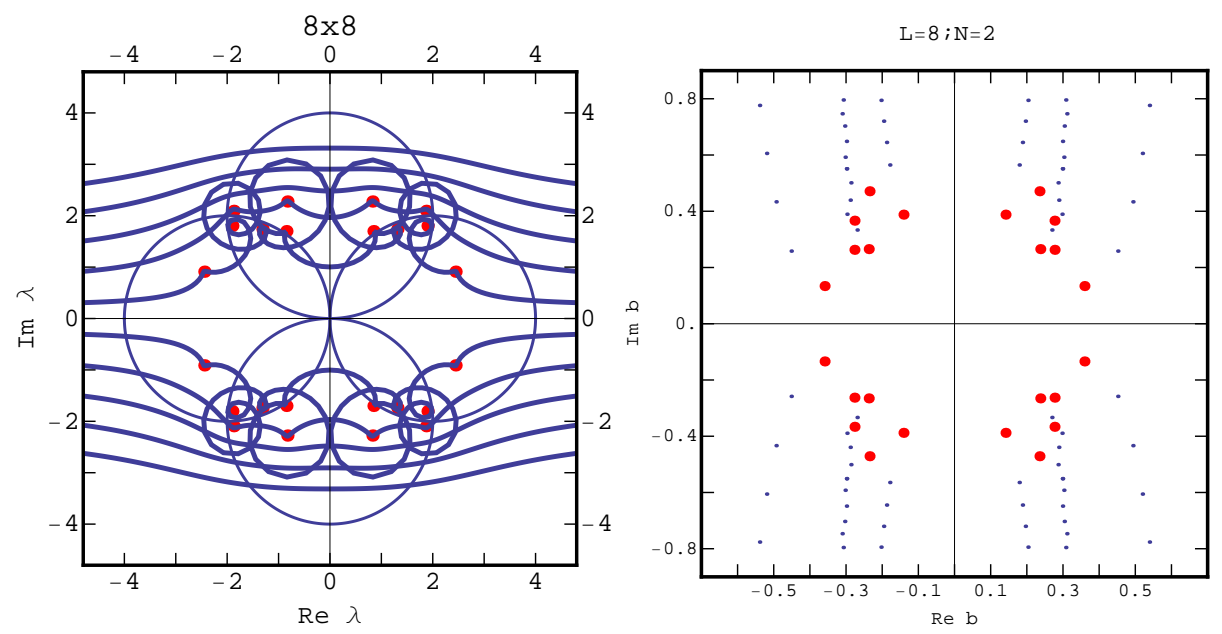

Figure 3: Left: images in the $\lambda^{t}$ plane of lines of constant imaginary part $2.25,1.75,1.25,0.75,0.25,-0.25$, ....,-2.25 in the complex mass gap plane and of the singular points (red dots) for a $8 \times 8$ lattice. Right: Fisher zeros for $N=2$ (blue) and images of singular points (red).

\section{4. $2-D O(N)$ nonlinear sigma models}

The nonlinear $O(N)$ sigma models on even cubic lattices have similar properties under the exchange of the sign of the coupling, namely $Z\left[-g^{2}\right]=\mathrm{e}^{4 D L^{D} / g^{2}} Z\left[g^{2}\right]$. The complex singularities of the average energy in 2 dimensions, for complex 't Hooft coupling $\lambda^{t}=g_{0}^{2} N$ have been studied in the large- $N$ limit . Details can be found in a recent publication [15]. A striking difference with the linear model is the absence of cut along the negative real axis. It was argued that the Fisher's zeros can only be inside a clover shaped region of the complex $\lambda^{t}$ plane or equivalently outside of a region delimited by 4 approximate hyperbolas with asymptotes on the boundary of a cross of width 0.5 centered at the origin in the $1 / \lambda^{t}$ plane. The argument holds for large $N$ and large volume. This limit is being studied using exact results at finite $N$ and finite volume. The graphs of Fig. 3 made with $N=2$ on a $8^{2}$ lattice can be compared with the corresponding ones in Ref. [15].

\section{Conclusions}

For pure gauge models with compact groups, there is no loss of vacuum when $g_{0}^{2} \rightarrow-g_{0}^{2}$, but only a change of vacuum. The discontinuity of the plaquette forbids the existence of a converging perturbative series but does not dictate the large order behavior. Reliable methods to locate Fisher's zeros are in progress. Non-perturbative effects should be accountable by modified expansions. New data for perturbative coefficients should help in this task .

\section{Acknowledgments}

This research was supported in part by the Department of Energy under Contract No. FG02-91ER40664. Y. Meurice thanks H. Perlt for valuables discussions on new data. 


\section{References}

[1] F. Dyson, Divergence of Perturbation Theory in Quantum Electrodynamics, Phys. Rev. 85631 (1952).

[2] J. C. LeGuillou and J. Zinn-Justin, Large-Order Behavior of Perturbation Theory. North Holland, Amsterdam, 1990.

[3] Y. Meurice, A simple method to make asymptotic series of Feynman diagrams converge, Phys. Rev. Lett. 88141601 (2002).

[4] B. Kessler, L. Li, and Y. Meurice, New optimization methods for converging perturbative series with a field cutoff, Phys. Rev. D69 045014 (2004).

[5] F. Di Renzo and L. Scorzato, A consistency check for renormalons in lattice gauge theory: $\beta^{-10}$ contributions to the su(3) plaquette, JHEP 10038 (2001).

[6] R. Horsley, P. E. L. Rakow, and G. Schierholz, Separating perturbative and non-perturbative contributions to the plaquette. Nucl. Phys. Proc. Suppl. 106870 (2002).

[7] Paul E. L. Rakow, Stochastic perturbation theory and the gluon condensate, PoSLAT2005 284 (2006).

[8] E. M. Ilgenfritz et al., Wilson loops in very high order lattice perturbation theory, arXiv:0910.2795, poster presented by H. Perlt at this conference .

[9] L. Li and Y. Meurice, About a possible 3rd order phase transition at $\mathrm{t}=0$ in $4 \mathrm{~d}$ gluodynamics, Phys. Rev. D73 036006 (2006).

[10] Wolfhard Janke and Ralph Kenna, Phase transition strengths from the density of partition function zeroes, Nucl. Phys. Proc. Suppl. 106905 (2002).

[11] Shinji Ejiri, Lee-Yang zero analysis for the study of QCD phase structure, Phys. Rev. D73 054502 (2006).

[12] A. Denbleyker, Daping Du, Yuzhi Liu, Y. Meurice, and A. Velytsky, Series expansions of the density of states in SU(2) lattice gauge theory, Phys. Rev. D78 054503 (2008).

[13] A. Bazavov and B. A. Berg, Heat bath efficiency with Metropolis-type updating, Phys. Rev. D 71 $114506(2005)$

[14] Alexei Bazavov and Bernd A. Berg, Program package for multicanonical simulations of U(1) lattice gauge theory, Comput. Phys. Commun. 1802339 (2009).

[15] Y. Meurice, Dyson instability for 2D nonlinear O(N) sigma models, Phys. Rev. D80 054020 (2009).

[16] L. Li and Y. Meurice, Lattice gluodynamics at negative $g^{2}$, Phys. Rev. D71 016008 (2005).

[17] L. Li and Y. Meurice, An example of optimal field cut in lattice gauge perturbation theory, Phys. Rev. D71 054509 (2005).

[18] Y. Meurice, The non-perturbative part of the plaquette in quenched QCD, Phys. Rev. D74 096005 (2006).

[19] Nelson A. Alves, Bernd A. Berg, and Sergiu Sanielevici, Spectral density study of the su(3) deconfining phase transition, Nucl. Phys. B376 218 (1992).

[20] A. Denbleyker, D. Du, and Y. Meurice, and A. Velytsky, Fisher's zeros of quasi-Gaussian densities of states, Phys. Rev. D76 116002 (2007). 